\title{
Hipoglicemia hiperinsulinêmica da infância
}

\author{
Hypoglycemia hypersinsulinemic of infancy
}

Raphael Del Roio Liberatore Junior', Carlos Eduardo Martinelli Junior ${ }^{2}$

\section{SUMÁRIO}

A hipoglicemia hiperinsulinêmica da infância (HHI) é uma emergência no período neonatal. Após curtos períodos de jejum, o cérebro ávido por glicose corre o risco de ficar sem seu principal substrato energético. Os critérios de diagnóstico de $\mathrm{HH}$, tanto no período neonatal quanto na criança maior e na adolescência, foram revisados. Foram descritas as etiologias e a fisiopatologia da HHI. As alterações moleculares frequentemente encontradas, bem como a descrição das principais mutações, são abordadas. Arq Bras Endocrinol Metab. 2011;55(3):177-83

\section{Descritores}

Hipoglicemia; insulina; hiperinsulinismo

\section{SUMMARY}

The hypoglycemia hyperinsulinemic of the infancy $(\mathrm{HHI})$ is an emergency in the neonatal period. After a short period of fast the avid brain runs out of its main energy substrate. The authors overhaul the diagnosis of $\mathrm{HH}$, not only in the neonatal period, but also in the late infant and in the adolescence. The aspects of the molecular alterations found in these cases, as well like the description of the main mutations are also approached. Arq Bras Endocrinol Metab. 2011;55(3):177-83

\section{Keywords}

Hypoglycemia; insulin; hiperinsulinism
1 Doutor em Pediatria, coordenador do Serviço de Endocrinologia Pediátrica, Departamento de Pediatria e Cirurgia Pediátrica, Faculdade de Medicina de São José do Rio Preto (Famerp), São José do Rio Preto, SP, Brasil 2 Livre-docente em Pediatria, coordenador do Serviço de Endocrinologia Pediátrica, Departamento de Pediatria e Puericultura, Famerp, São José do Rio Preto, SP, Brasil

Correspondência para:

Raphael Del Roio Liberatore Junior Rua Ondina, 54

15015-205 - São José do Rio Preto, SP, Brasil

liberat@famerp.br

Recebido em 10/Fev/2011 Aceito em 18/Abr/2011

\section{INTRODUÇÃO}

$\mathrm{A}^{\mathrm{n}}$ manutenção de adequados níveis glicêmicos é de vital importância em todas as idades, mas é particularmente crucial no período neonatal e na lactância $(1,2)$.

Nesses períodos, o tecido cerebral ainda não completamente maduro, marcado por intensa atividade metabólica e extremamente ávido por glicose, torna-se bastante sensível às reduções de níveis glicêmicos mesmo que fugazes.

Dessa forma, a ocorrência de episódios repetidos de hipoglicemia nessa fase da vida pode trazer danos cerebrais, na maioria das vezes graves e irreversíveis (1-4).

A secreção inapropriadamente aumentada de insulina, sem relação com os níveis de glicemia, caracteriza hiperinsulinemia, que é a principal causa de hipoglicemia persistente e recorrente nessa fase da vida $(1,5,6)$.

Essa situação clínica é marcada pelo aumento da utilização da glicose pelos tecidos sensíveis às ações da insulina, ao mesmo tempo que o aumento da concentração de insulina inibe a produção endógena de glicose, bloqueando a glicólise e a neoglicogênese. Em decorrência desse bloqueio, não ocorre produção de corpos cetônicos, substrato energético alternativo para o tecido cerebral (6).

A queda acentuada dos níveis glicêmicos e a falta de produção de corpos cetônicos são as causas dos danos cerebrais frequentemente associados a essa condição clínica. $\mathrm{O}$ cérebro em desenvolvimento ávido por combustível não tem como utilizar nenhuma fonte energética. Assim, essa situação clínica se transforma em uma das emergências do período neonatal.

A identificação precoce e a correta abordagem da hipoglicemia hiperinsulinêmica $(\mathrm{HH})$ são, dessa forma, de extrema importância para reduzir a morbi-mortalidade dessa patologia.

\section{ETIOLOGIAS}

Várias são as causas da HH. Essas podem ser congênitas, secundárias, associadas a síndromes genéticas ou associadas a doenças metabólicas $(1,3,6)$. 
As formas secundárias no período neonatal são normalmente transitórias e se associam a presença de diabetes materno, asfixia ou incompatibilidade $\mathrm{Rh}$.

Várias síndromes genéticas podem estar associadas à $\mathrm{HH}$, tais como Beckwith-Wiedemann, Soto, Trissomia do 13, Turner mosaico e outras. Dessas, a mais frequente é a síndrome de Beckwith-Wiedemann, na qual ocorre a associação de $\mathrm{HH}$, hemi-hipertrofia, defeitos da parede abdominal, macroglossia e alterações das orelhas.

As desordens congênitas da glicosilação $(C D G)$ e a tirosinemia tipo 1 são as doenças metabólicas que cursam com $\mathrm{HH}$. As formas congênitas de $\mathrm{HH}$ (HHC) constituem um grupo bastante heterogêneo e são as formas mais graves e mais frequentes no período neonatal. As HHC podem se apresentar com variadas características clínicas e subgrupos histológicos. As características moleculares, foco de estudos mais recentes, permitem uma melhor avaliação da possível distribuição, difusa ou focal, da lesão pancreática (6-8).

A tabela $\mathrm{l}$ mostra as principais causas de $\mathrm{HH}$ e seus quadros clínicos resumidos.

Tabela 1. Causas de hipoglicemia hiperinsulinêmica

\begin{tabular}{l}
\hline Hiperinsulinemia congênita (herança) \\
ABCC8 (autossômica recessiva e dominante) \\
KCNJ11 (autossômica recessiva e dominante) \\
GLUD1 (dominante) \\
GCK (dominante) \\
HADH (recessiva) \\
HNF4A (dominante) \\
SLC16A1 (induzida pelo exercício) (dominante) \\
Secundária (normalmente transitória) \\
Diabetes melito materno (gestational ou insulinodependente) \\
Restrição de crescimento intrauterino \\
Asfixia perinatal \\
Isoimunização Rh \\
Causas metabólicas \\
Doenças congênitas da glicosilação (CDG), Tipo 1a/b/d \\
Tirosinemia tipo I \\
Associadas a síndromes \\
Beckwith-Wiedemann \\
Soto \\
Kabuki \\
Usher \\
Timothy \\
Costello \\
Trisomy 13 \\
Turner mosaico \\
Outras \\
Síndrome "Dumping" \\
Insulinoma (esporádico ou associado a MEN 1) \\
Mutações do gene do receptor da insulina \\
"Munchausen-by-proxy" \\
\hline Fonte: Modificada de Kapoor RR e cols. (9). \\
\hline
\end{tabular}

\section{APRESENTAÇÃO CLÍNICA}

Os sinais e sintomas de hipoglicemia são os marcadores clínicos da $\mathrm{HH}$ e podem ocorrer em qualquer faixa etária.

No período neonatal, esses sinais e sintomas são mais inespecíficos e a possibilidade de hipoglicemia deve ser sempre considerada na vigência de palidez, má-perfusão tecidual, letargia, falta de sucção ou convulsões. Macrossomia, embora não esteja presente em todos os casos de $\mathrm{HH}$ neonatal, pode ser observada em alguns casos como resultado dos níveis elevados de insulina no período intrauterino. A paradoxal dificuldade de alimentação em situação de hipoglicemia, observada em grande número de pacientes e muitas vezes atribuída a lesões de sistema nervoso central secundárias aos episódios de hipoglicemias, pode ser explicada pela saciedade induzida pela hiperinsulinemia e grelina $(1,10)$.

Em crianças maiores e adolescentes, sinais e sintomas de neuroglicopenia, tais como sonolência, alterações de comportamento, cefaleia, torpor, tremor, convulsões e coma, normalmente estão associados a sinais e sintomas autonômicos, como sudorese fria, taquicardia e palidez (1-6).

O quadro clínico se instala rapidamente após um período curto de jejum ou após atividade física e a hipoglicemia habitualmente não é corrigida somente com alimentação por via oral, havendo a necessidade de infusão de grandes aportes de glicose endovenosa.

\section{DIAGNÓSTICO}

Níveis glicêmicos anormalmente baixos, em episódios repetidos e que necessitam de infusão endovenosa de glicose, são características bastante sugestivas de $\mathrm{HH}$ e devem ser investigados como tal.

A necessidade de infusão contínua de glicose em concentrações maiores do que $10 \mathrm{mg} / \mathrm{kg} /$ minuto para a manutenção da glicemia e a dependência do aporte de glicose endovenosa são marcadores de $\mathrm{HH}(1,10)$.

Durante o episódio de hipoglicemia, que pode ser espontâneo ou provocado pelo jejum, a confirmação diagnóstica da $\mathrm{HH}$ necessita da coleta de exames adicionais conhecidos como "amostra crítica" pela maioria dos autores.

A amostra crítica é a coleta de amostras para dosagem sérica de glicose, cetonas, ácidos graxos, gasometria ( $\mathrm{pH}$ e bicarbonato), lactato, piruvato, amônia, hormônio de crescimento, cortisol e insulina e/ou peptídeo $\mathrm{C}$, além da pesquisa para erros inatos de metabolismo. 
Nos casos altamente sugestivos de $\mathrm{HH}$, não há a necessidade da realização de todos os exames ao mesmo tempo e as amostras podem ser estocadas para posterior análise, se necessário.

$\mathrm{O}$ diagnóstico laboratorial de $\mathrm{HH}$ compreende a documentação de concentrações detectáveis de insulina na vigência de hipoglicemia, associada à cetonemia negativa e concentrações reduzidas de ácidos graxos circulantes. Por vezes, somente uma dosagem não é suficiente para a conclusão diagnóstica e, na dúvida, a necessidade de altas taxas de infusão endovenosa de glicose deve dirigir o diagnóstico $(1,6)$.

\section{FISIOPATOLOGIA}

Com relação à fisiopatologia, a $\mathrm{HH}$ pode didaticamente ser dividida em "canelopatias" ou "metabolitopatias", quando envolvem respectivamente defeitos nos canais das células B pancreáticas ou quando ocorre acúmulo de metabólitos intermediários que desencadeiam a secreção de insulina.

Nas células B pancreáticas, os canais de potássio sensíveis à adenosina-trifosfato (canal $\mathrm{K}_{\mathrm{ATP}}$ ) exercem papel fundamental no controle da síntese de insulina estimulada pela glicose.

Os canais $\mathrm{K}_{\text {ATP }}$ são complexos proteicos heterooctaméricos transmembrana e compostos de quatro canais retificadores de potássio (Kir6.2) e 4 receptores 1 de sulfonilureia (SURI). Quando a glicose se acopla ao canal $\mathrm{K}_{\mathrm{ATP}}$, leva ao aumento da relação ATP/ADP intracelular e fechamento desses canais e consequente despolarização da membrana celular.

A despolarização provoca a abertura dos canais de cálcio levando ao influxo de cálcio. Esse influxo eleva a concentração de cálcio intracelular, que é o passo final para a exostose dos grânulos de insulina.

Quando ocorrem alterações dos canais $\mathrm{K}_{\mathrm{ATP}}$, a célula $B$ perde a sensibilidade na regulação do balanço ATP/ ADP e, dessa forma, provoca o fechamento anormal dos canais e consequente secreção inadequada de insulina.

A figura 1 mostra esquematicamente a célula B e os canais de potássio, bem como a exocitose de insulina.

Defeitos desses canais podem resultar em secreção deficiente ou secreção aumentada de insulina, resultando, respectivamente, em quadros de diabetes melito ou de $\mathrm{HH}(11-20)$.

Quanto às metabolitopatias, os defeitos podem envolver três enzimas reguladoras da síntese de insulina:

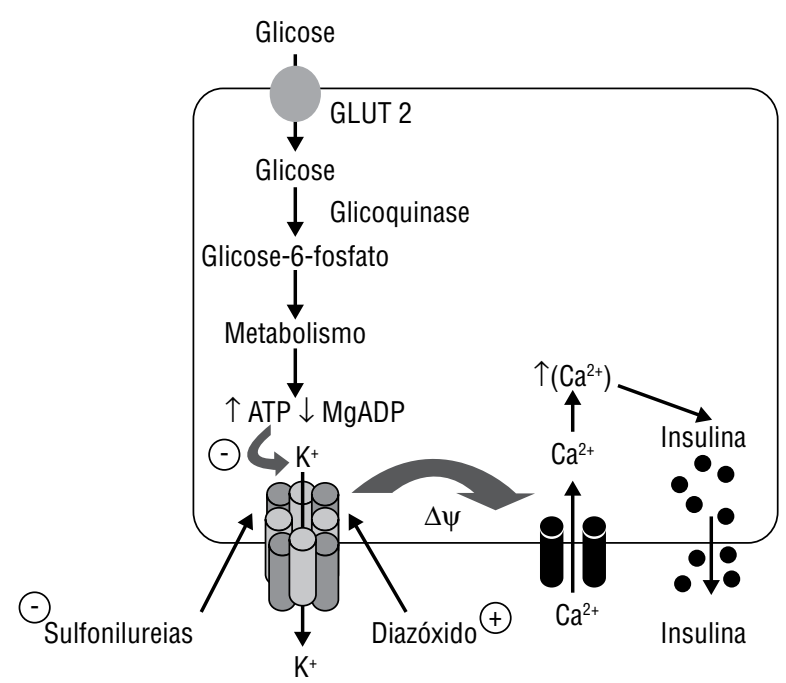

Figura 1. Representação esquemática da célula B pancreática e o mecanismo de secreção de insulina. Fonte: Modificado de Flanagan SE (21).

glutamato de-hidrogenase, glicoquinase e hidroxiacil-CoA-de-hidrogenase de cadeia curta (short-chain 3-hydroxyacyl-CoA dehydrogenases - SCHD).

As mutações ativadoras da enzima glutamato de-hidrogenase são a segunda causa mais frequente de HH congênita. Nesses casos, conhecidos antigamente como HH sensível à leucina, ocorre elevação dos níveis de amônia associado à $\mathrm{HH}$, sem, no entanto, existir clínica de hiperamonemia (letargia, cefaleia). A enzima glutamato de-hidrogenase controla a produção de GTP e consequente elevação dos níveis de ATP. A mutação da glutamato de-hidrogenase leva ao aumento da relação ATP/ADP no interior da célula B pancreática, pois a enzima se torna mais sensível aos níveis de leucina, aumentando a oxidação de glutamato para GTP, elevando os níveis de ATP e desencadeando o fechamento do canal $\mathrm{K}_{\mathrm{ATP}}$, liberando insulina. Nesses casos, a hipoglicemia pode ocorrer tanto após a ingestão de leucina quanto após um período de jejum, em recém-natos, mas também em crianças mais velhas. Tipicamente, essas crianças apresentam convulsões do tipo ausência associadas a alterações eletroencefalográficas de padrão de epilepsia generalizada (22-26).

A enzima glicoquinase é importante como sensor celular do nível de glicose. Quanto maior é o nível de glicose, maior é a atividade dessa enzima, elevando a transformação da glicose para glicose-6-fosfato e consequente aumento de ATP. As mutações que causam ativação dessa enzima reduzem o nível de disparo da secreção de insulina mediada pelo nível glicêmico, dessa forma liberando insulina mesmo sob níveis baixos de 
glicose. Os portadores desse tipo de defeito sofrem de HH sensível ao diazóxido, na maioria das vezes. Recentemente foi descrito um portador de $\mathrm{HH}$ severa e não responsiva ao diazóxido e que apresentava uma mutação "de novo" no gene da glicoquinase (22-26).

A deficiência da SCHD é a causa ainda de mecanismo não totalmente explicado de $\mathrm{HH}$. Nesses casos, formas graves no período neonatal e formas mais brandas em idades mais avançadas podem se apresentar. O mecanismo pelo qual alterações dessa enzima podem levar a quadros de $\mathrm{HH}$ ainda não está determinado $(25,26)$.

\section{HISTOLOGIA}

Duas formas histológicas se apresentam nos casos de $\mathrm{HH}$, ambas com aumento no volume celular das células B pancreáticas, representando uma maior atividade biológica e conhecida como adenomatose pancreática.

A forma difusa, conhecida como Nesidioblastose, apresenta todas as células B com o padrão histológico acima descrito. Pode ser familiar ou esporádica e ser produto de mutação de transmissão recessiva ou dominante $(1,6)$.

A forma focal apresenta somente pequenas regiões do pâncreas com adenomatose, em geral medindo de 2 a $10 \mathrm{~mm}$. Esses casos são normalmente esporádicos $(1,6)$.

A incidência das formas esporádicas varia de 1:40.000 a 1:50.000 nascidos vivos. Nas formas familiares, essa incidência pode chegar a 1 caso a cada 2.500 nascimentos $(1,6)$.

\section{GENÉTICA DO HIPERINSULINISMO CONGÊNITO}

Mutações em sete diferentes genes são responsáveis por cerca de $50 \%$ dos casos de HHC. Os sete genes envolvidos são: ABCC8, KCNJ11, GLUD1, CGK, HADH, SLC16Al e HNF4A. O restante dos casos ainda permanece com etiologias desconhecidas, mas a descrição de novas mutações e/ou novos genes envolvidos deverá resultar na elucidação de outros casos.

\section{ABCC8 e KCNJ11}

Esses dois genes, ABCC8 e KCNJl, localizados em regiões vizinhas do cromossomo 11 (1lpl5.1) são os responsáveis, respectivamente, pelas proteínas SURI e
Kir6.2 e, assim, desempenham papel importante no controle da secreção de insulina pela célula B pancreática.

As mutações inativadoras nesses genes são responsáveis pelas formas mais comuns e mais graves de $\mathrm{HH}$. Nesses casos, os portadores de $\mathrm{HH}$ da forma difusa não respondem ao tratamento medicamentoso e necessitam de pancreatectomia subtotal para controle dos níveis glicêmicos. Tratam-se, na maioria das vezes, de mutações de transmissão recessiva, podendo eventualmente apresentar herança dominante.

As mutações de transmissão autossômico-dominantes são responsáveis por formas mais leves, focais, sob o ponto de vista histológico e inclusive responsíveis ao tratamento medicamentoso (26-28).

As mutações encontradas nesses dois genes são normalmente responsáveis por cerca de $50 \%$ dos casos com etiologia genética determinada de $\mathrm{HH}$. Entretanto, em japoneses, somente $20 \%$ dos casos descritos envolvem mutações desses genes.

\section{GLUD1 e GCK}

GLUDl e GCK são responsáveis por codificar, respectivamente, as enzimas glutamato de-hidrogenase e glucoquinase.

As mutações ativadoras de GLUDl (10q23.3) levam a síndrome de $\mathrm{HH}$ e hiperamonemia, que é a segunda forma mais frequente de HH. A ativação dessa enzima aumenta a oxidação de glutamato e aumenta a relação ATP/ADP na célula $\mathrm{B}$. As mutações ativadoras de GCK (7pl5-pl3) reduzem o limite da secreção de insulina estimulada pela glicose (29-41).

Todas as mutações descritas até o momento nesses dois genes apresentaram transmissão dominante e a forma histológica descrita é a forma difusa e sem resposta ao tratamento medicamentoso.

\section{HADH}

O gene HADH (4q22-q26) é responsável por codificar a enzima L-3 hidroxacil coenzima A de-hidrogenase, enzima mitocondrial que catalisa a fase final de oxidação de ácidos graxos. O mecanismo de ação dessa enzima no controle de funcionamento da célula $B$ não é ainda conhecido. Mutações inativadoras de $\mathrm{HADH}$ foram descritas em portadores de $\mathrm{HH}(21,42)$.

A forma de transmissão descrita é a recessiva e a forma histológica encontrada, a difusa. Clinicamente, esses casos parecem não responder ao tratamento medicamentoso. 


\section{SLC16A1}

O gene SLCl6Al (lpl3.2-pl2) codifica uma proteína transmembrana denominada MCTl (transportador 1 monocarboxilado). A função dessa enzima não está ainda bem definida.

A transmissão das mutações do SLCl6Al ocorre com padrão dominante e a forma histológica descrita é a forma difusa. O quadro clínico envolve o desenvolvimento de hipoglicemia induzida pelo exercício físico anaeróbico $(9,43-45)$.

\section{HNF4A}

Mutações no gene HNF4A (20q12-q13.1) que codifica a proteína fator 4 do núcleo de hepatócito podem causar formas transitórias ou persistentes de $\mathrm{HH}$. Essa proteína parece exercer um controle funcional da atuação da célula $\mathrm{B}$, em um mecanismo ainda não completamente conhecido.

Mutações em heterozigose foram descritas como causadoras de $\mathrm{HH}$ associadas à macrossomia, com mecanismo ainda desconhecido. A forma de transmissão descrita é a dominante e a forma histológica, a difusa $(9,43-45)$.

A HH é uma doença grave de alta morbi-mortalidade. Em cerca de metade dos casos está envolvida etiologia genética, de forma que mutações em sete diferentes genes promovem diferentes quadros clínicos, histológicos e com diferentes respostas ao tratamento medicamentoso.

A correta determinação do gene envolvido permite inferir o tipo histológico e a resposta ao tratamento medicamentoso ou, em outras palavras, a necessidade ou não de se realizar pancreatectomia com urgência.

\section{TRATAMENTO}

\section{Tratamento de suporte}

As crianças com HHI devem ser encaradas como gravemente enfermas e tratadas como tal.

O risco de desenvolvimento de sequelas neurológicas e o risco de desenvolver sobrecarga cardiovascular por excesso de volume devem estar presentes na memória do médico-assistente (2).

Crianças com HHI frequentemente são também portadoras de doença do refluxo gastroesofágico e também frequentemente são anoréticas a despeito dos elevados níveis de insulina circulantes.
A opção pela utilização de sondas oro ou nasogástricas deve ser cuidadosamente pensada e jamais adotada sem a correta abordagem medicamentosa desses casos.

Por outro lado, é fundamental um acesso venoso seguro, suficientemente calibroso e de preferência uma linha venosa central deve ser instalada. Deve-se optar, nesses casos, por cateteres de demora, instalados eventualmente por via cirúrgica. Dissecção venosa não é a primeira indicação para tais casos.

Essa via endovenosa confiável previne o desenvolvimento de hipoglicemia de rebote, após a suspensão periódica da infusão de glicose, por perda de acesso periférico ou para infusão de outros medicamentos. Também a necessidade de altas concentrações de glicose, inclusive pela necessidade do controle do volume oferecido, pode causar flebite em veias periféricas.

Dessa forma, o quanto antes, acesso central confiável deve ser providenciado. Essa linha venosa deve ser reservada apenas para infusão de glicose e todo cuidado deve ser tomado para prevenir infecção desse cateter continuamente banhado com líquido altamente "açucarado".

\section{Abordagem na urgência}

O nível glicêmico deve ser corrigido o mais rapidamente possível, principalmente no lactente pequeno.

A medicação de escolha deve obviamente ser uma solução glicosada, preferencialmente em concentração tanto mais baixa quanto menor for a criança.

Quando a situação clínica não permitir a infusão endovenosa, a solução glicosada pode ser infundida por via intraóssea ou subcutânea.

Imediatamente após a infusão aguda de glicose, é de fundamental importância a manutenção do aporte de glicose para manutenção dos níveis glicêmicos.

Também quando a situação clínica impedir a rápida obtenção de uma via endovenosa, pode ser usado Glucagon por via subcutânea, na dose de 0,5 a 1,0 mg. Essa medicação só apresenta resposta quando a criança tiver boa reserva de glicogênio e permite que alguns poucos minutos de normoglicemia sejam utilizados para conseguir acesso venoso.

\section{Tratamento inicial}

Após a instalação de infusão com glicose, deve-se fornecer a quantidade necessária para manter níveis glicêmicos próximos de $100 \mathrm{mg} / \mathrm{dl}$, em concentração adequada para evitar sobrecarga de volume.

Em veia central, concentrações de até $50 \%$ podem ser utilizadas. 
Concomitantemente, também infusão endovenosa ou subcutânea contínua de Glucagon, com doses variando entre 1 e $20 \mathrm{ug} / \mathrm{kg} /$ hora, pode ser utilizada na tentativa de se utilizar menores concentrações de glicose, principalmente por veia periférica $(9,43-45)$.

Voltamos a ressaltar a necessidade da linha venosa central o quanto antes.

\section{Tratamento medicamentoso após a urgência}

A droga de escolha nos casos de HHI é o diazóxido. Essa medicação se liga aos canais $\mathrm{K}_{\mathrm{ATP}}$ e os mantêm abertos evitando a despolarização da membrana e consequente secreção de insulina pelas células B pancreáticas $(9,43-45)$.

Com exceção dos portadores de mutações inativadoras dos genes ABCC8 ou KCNJ11 e dos portadores das formas focais de HHI, todas as crianças com HHI respondem ao uso de diazóxido. A dose utilizada varia de 5 a $20 \mathrm{mg} / \mathrm{kg} /$ dia, dividida em três tomadas a cada 8 horas, por via oral.

Trata-se de um potente retentor hídrico e pode também provocar leucopenia, eosinofilia e hipertricose.

Deve ser usado associado a clorotiazida, na dose de 7 a $10 \mathrm{mg} / \mathrm{kg} /$ dia, dividida em duas tomadas a cada 12 horas, por via oral. O tiazídico, além de proteger contra a sobrecarga de volume, também possui efeito hiperglicemiante e, dessa forma, age em sinergismo ao diazóxido. Pode provocar hiponatremia e hipocalemia e, desse modo, os níveis séricos desses eletrólitos devem ser periodicamente monitorizados $(9,43-45)$.

Mesmo durante a utilização concomitante de diurético, extrema atenção deve ser dada à sobrecarga hídrica e eventualmente restrição de volume pode ser necessária.

Devem-se manter doses suficientes das duas medicações para retirar o aporte endovenoso. Retirar a linha endovenosa após a completa estabilização do quadro, sem necessidade de medicação endovenosa.

Naquelas crianças que não respondem ao diazóxido, é fundamental a determinação de mutações nos genes ABCC8 e KCNJ11 para distinção entre as formas focais e difusas da HHI (2).

Até que a análise genética seja efetuada, pode ser tentada infusão contínua de Octreotide por via subcutânea. As doses variam entre 5 e $30 \mathrm{ug} / \mathrm{kg} /$ dia. De forma intermitente, pode ser administrado sob via subcutânea a cada 6 ou 8 horas $(9,43-45)$.

Em nosso meio, não existe a possibilidade da determinação da lesão focal e, dessa forma, nos casos resis- tentes ao tratamento medicamentoso a terapia de escolha é a pancreatectomia subtotal. No intraoperatório, imediatamente após a interrupção do fluxo sanguíneo ao pâncreas, deve-se iniciar a redução da infusão das drogas hiperglicemiantes e do aporte de glicose. A utilização de métodos de imagem, mesmo no intraoperatório, não permite localizar lesões focais (46).

É alta a incidência de insuficiência exócrina e diabetes melito no seguimento dessas crianças.

Declaração: os autores declaram não haver conflitos de interesse científico neste estudo.

\section{REFERÊNCIAS}

1. Stanley CA. Hyperinsulinism in infants and children. Pediatr Clin North Am. 1997;44:363-74.

2. Hussain K, Balnkestein O, De Lonlay P, Christesen HT. Hyperinsulinemic hypoglycaemia: biochemical basis and the importance of maintaining normoglycaemia during management. Arch Dis Child. 2007;92:598-70.

3. Bruninig GJ. Recent advances in hyperinsulinism and the pathogenesis of diabetes mellitus. Curr Opin Pediatr. 1990;2:758-65.

4. Fournet JC, Junien C. The genetics of neonatal hyperinsulinism. Horm Res. 2003;59:30-4.

5. Glaser B, Thornton PS, Otonkoski T, Junien C. The genetics of neonatal hyperinsulinism. Arch Dis Child. 2000;82:79-86.

6. de Lonlay P, Fournet JC, Touati G, Groos MS, Martin D, Sevin C, et al. Heterogeneity of persistent hyperinsulinaemic hypoglycaemia. A series of 175 cases. Eur J Pediatr. 2002;161:37-48.

7. Meissner T, Mayatepek E. Clinical and genetic heterogeneity in congenital hyperinsulinism. Eur J Pediatr. 2002;161:6-20.

8. Sempoux C, Guiot $Y$, Lefevre A, Nihoul-Fekete C, Jaubert F, Saudubray JM, et al. Neonatal hyperinsulinemic hypoglycemia: heterogeneity of the syndrome and keys for differential diagnosis. J Clin Endocrinol Metab. 1998;83:1455-61.

9. Kapoor RR, James C, Hussain K. Advances in the diagnosis and management of hyperinsulinemic hypoglycemia. Nat Clin Pract Endocrinol Metab. 2009;5:101-12.

10. Karczewska-Kupczewska M, Straczkowski M, Adamska A, Nikolajuk A, Otziomek E, Górska M, et al. Increased suppression of serum ghrelin concentration by hyperinsulinemia in women with anorexia nervosa. Eur J Endocrinol. 2010;162(2):235-9.

11. Nestorowicz A, Glaser B, Wilson BA, Shyng SL, Nichols CG, Stanley $C A$, et al. Genetic heterogeneity in familial hyperinsulinism. Hum Mol Genet. 1998;7:1119-28.

12. Thomas $P, Y e Y$, Lightner E. Mutation of the pancreatic islet inward rectifier Kir6.2 also leads to familial persistent hyperinsulinemic hypoglycemia of infancy. Hum Mol Genet. 1996;5:1809-12.

13. Thomas PM, Cote GJ, Wohllk N, Haddad B, Mathew PM, Rabl W, et al. Mutations in the sulfonylurea receptor gene in familial persistent hyperinsulinemic hypoglycemia of infancy. Science. 1995;268:426-9.

14. Nestorowicz A, Inagaki N, Gonoi T, Schoor KP, Wilson BA, Glaser $B$, et al. A nonsense mutation in the inward rectifier potassium channel gene, Kir6.2, is associated with familial hyperinsulinism. Diabetes. 1997;46:1743-8.

15. Dunne MJ, Kane C, Shepherd RM, Sanchez JA, James RF, Johnson PR, et al. Familial persistent hyperinsulinemic hypoglycemia of infancy and mutations in the sulfonylurea receptor. $\mathrm{N}$ Engl J Med. 1997;336:703-6. 
16. OtonkoskiT, Ammala C, Huopio H, Cote GJ, Chapman J, Cosgrove K, et al. A point mutation inactivating the sulfonylurea receptor causes the severe form of persistent hyperinsulinemic hypoglycemia of infancy in Finland. Diabetes. 1999;48:408-15.

17. Tanizawa Y, Matsuda K, Matsuo M, Ohta Y, Ochi N, Adachi M, et al. Genetic analysis of Japanese patients with persistent hyperinsulinemic hypoglycemia of infancy: nucleotide-binding fold-2 mutation impairs cooperative binding of adenine nucleotides to sulfonylurea receptor 1. Diabetes. 2000;49:114-20.

18. Marthinet E, Bloc A, Oka Y, Tanizawa Y, Wehrle-Haller B, Bancila V, et al. Severe congenital hyperinsulinism caused by a mutation in the Kir6. 2 subunit of the adenosine triphosphate-sensitive potassium channel impairing trafficking and function. J Clin Endocrinol Metab. 2005;90:5401-6.

19. Tornovsky S, Crane A, Cosgrove KE, Hussain K, Lavie J, Heyman $M$, et al. Hyperinsulinism of infancy: novel ABCC8 and KCNJ11 mutations and evidence for additional locus heterogeneity. J Clin Endocrinol Metab. 2004;89:6224-34.

20. Ashcroft FM, Harrison DE, Ashcroft SJ. Glucose induces closure of single potassium channels in isolated rat pancreatic beta-cells. Nature. 1984;312:446-8.

21. Flanagan $S E$, Clauin $S$, Bellanne-Chantelot $C$, Lonlay $P$, Harries $\mathrm{LW}$, Gloyn AL, et al. Update of mutations in the genes encoding the pancreatic beta-cell K(ATP) channel subunits Kir6.2 (KCNJ11) and sulfonylurea receptor 1 (ABCC8) in diabetes mellitus and hyperinsulinism. Human Mutation. 2009;30:170-80.

22. Clayton PT, Eaton S, Aynsley-Green A, Edginton M, Hussain K, Krywawych $S$, et al. Hyperinsulinism in short-chain L-3-hydroxyacyl-CoA dehydrogenase deficiency reveals the importance of beta-oxidation in insulin secretion. J Clin Invest. 2001;108:457-65.

23. Hussain K, Clayton PT, Krywawych S, Chatziandreou I, Mills P, Ginbey DW, et al. Hyperinsulinism of infancy associated with a novel splice site mutation in the SCHAD gene. J Pediatr. 2005;146:706-8.

24. Molven A, Matre GE, Duran M, Wanders RJ, Rishaug U, Njolstad $\mathrm{PR}$, et al. Familial hyperinsulinaemic hypoglycemia caused by a defect in the SCHAD enzyme of mitochondrial fatty acid oxidation. Diabetes. 2004;53:221-7.

25. Huopio H, Reimann F, Ashfield R, Komulainen J, Lenko HL, Rahier $\mathrm{J}$, et al. Dominantly inherited hyperinsulinism caused by a mutation in the sulfonylurea receptor type 1 . J Clin Invest. 2000;106:897-906.

26. Thornton PS, MacMullen C, Ganguly A, Ruchelli E, Steinkrauss L, Crane A, et al. Clinical and molecular characterization of a dominant form of congenital hyperinsulinism caused by a mutation in the high-affinity sulfonylurea receptor. Diabetes. 2003;52:2403-10.

27. Pinney SE, MacMullen C, Becker S, Lin YW, Hanna C, Thornton P, et al. Clinical characteristics and biochemical mechanisms of congenital hyperinsulinism associated with dominant KATP channel mutations. J Clin Invest. 2008;118:2877-86.

28. Lin YW, Macmullen C, Ganguly A, Stanley CA, Shyng SL. A novel KCNJ11 mutation associated with congenital hyperinsulinism reduces the intrinsic open probability of beta-cell ATP-sensitive potassium channels. J Biol Chem. 2006;281:3006-12.

29. Stanley CA, Lieu YK, Hsu BYL, Burlina AB, Greenberg CR, Hopwood $\mathrm{NJ}$, et al. Hyperinsulinism and hyperammonemia in infants with regulatory mutations of the glutamate dehydrogenase gene. N Engl J Med. 1998;338:1352-7.

30. Stanley CA, Fang J, Kutyna K, Hsu BY, Ming JE, Glaser B, et al. Molecular basis and characterization of the hyperinsulinism/ hyperammonemia syndrome: predominance of mutations in exons 11 and 12 of the glutamate dehydrogenase gene. Diabetes. 2000;49:667-73.

31. Yorifuji T, Muroi J, Uematsu A, Hiramatsu H, MomoiT. Hyperinsulinism hyperammonemia syndrome caused by mutant glutamate dehydrogenase accompanied by novel enzyme kinetics. Hum Genet. 1999;104:476-9.

32. Miki $Y$, Taki T, Ohura T, Kato H, Yanagisawa M, Hayashi Y. Novel missense mutations in the glutamate dehydrogenase gene in the congenital hyperinsulinism hyperammonemia syndrome. J Pediatr. 2000;136:69-72.

33. Santer R, Kinner M, Passarge $M$, Superti-Furga $A$, Mayatepek $E$, MeissnerT, et al. Novel missense mutations outside the allosteric domain of glutamate dehydrogenase are prevalent in European patients with the congenital hyperinsulinism-hyperammonemia syndrome. Hum Genet. 2001;108:66-71.

34. Fujioka $\mathrm{H}$, Okano $\mathrm{Y}$, Inada $\mathrm{H}$, Asada $\mathrm{M}$, Kawamura T, Hase $\mathrm{Y}$, et al. Molecular characterisation of glutamate dehydrogenase gene defects in Japanese patients with congenital hyperinsulinism/hyperammonaemia. Eur J Hum Genet. 2001;9:931-7.

35. Glaser B, Kesavan P, Heyman M, Davis E, Cuesta A, Buchs A, et al. Familial hyperinsulinism caused by an activating glucokinase mutation. N Engl J Med. 1998;338:226-30.

36. Christesen HB, Jacobsen BB, Odili S, Buettger C, Cuesta-Munoz A, Hansen $T$, et al. The second activating glucokinase mutation (A456V): implications for glucose homeostasis and diabetes therapy. Diabetes. 2002;51:1240-6.

37. Gloyn AL, Noordam K, Willemsen MA, Ellard S, Lam WW, Campbell IW, et al. Insights into the biochemical and genetic basis of glucokinase activation from naturally occurring hypoglycemia mutations. Diabetes. 2003;52:2433-40.

38. Cuesta-Munoz AL, Huopio H, Otonkoski T, Gomez-Zumaquero JM, Nanto-Salonen K, Rahier J, et al. Severe persistent hyperinsulinemic hypoglycemia due to a de novo glucokinase mutation. Diabetes. 2004;53:2164-8.

39. Dullaart RP, Hoogenberg K, Rouwe CW, Stulp BK. Family with autosomal dominant hyperinsulinism associated with $\mathrm{A} 456 \mathrm{~V}$ mutation in the glucokinase gene. J Intern Med. 2004;255:143-5.

40. Wabitsch M, Lahr G, Van de Bunt M, Marchant C, Lindner M, von Puttkamer $\mathrm{J}$, et al. Heterogeneity in disease severity in a family with a novel G68V GCK activating mutation causing persistent hyperinsulinaemic hypoglycaemia of infancy. Diabet Med. 2007;24:1393-9.

41. Christesen HB, Tribble ND, Molven A, Siddiqui J, Sandal T, Brusgaard $\mathrm{K}$, et al. Activating glucokinase (GCK) mutations as a cause of medically responsive congenital hyperinsulinism: prevalence in children and characterisation of a novel GCK mutation. Eur J Endocrinol. 2008;159:27-34.

42. James C, Kapoor RR, Ismail D, Hussain K. The genetic basis of congenital hyperinsulinism. J Med Genet. 2009;46:289-99.

43. Flanagan SE, Kapoor RR, Mali G, Cody D, Murphy N, Schwahn B, et al. Diazoxide-responsive hyperinsulinemic hypoglycemia caused by HNF4A gene mutations. Eur J Endocrinol. 2010;162:987-92.

44. Hussain K. Diagnosis and management of hyperinsulinaemic hypoglycaemia of infancy. Horm Res. 2008;69:2-13.

45. Kapoor RR, Flanagan SE, James C, Shield JP, Ellard S, et al. Hyperinsulinaemic hypoglycaemia. Arch Dis Child. 2009;94:450-7.

46. Liberatore Jr RDR, Vieira EM, Ferreira RHG, Onate G. Anestesia em criança com nesidioblastose. Rev Bras Anest. 2000;50:45. 